\title{
First-Principle Studies on the Ga and As Doping of Germanane Monolayer
}

\author{
Lei Liu, Yanju Ji*, Yifan Liu, Liqiang Liu \\ School of Science, Shandong Jianzhu University, Jinan, China \\ Email: ^2922977166@qq.com
}

How to cite this paper: Liu, L., Ji, Y.J., Liu, Y.F. and Liu, L.Q. (2019) First-Principle Studies on the Ga and As Doping of Germanane Monolayer. Journal of Applied Mathematics and Physics, 7, 46-54. https://doi.org/10.4236/jamp.2019.71005

Received: November 21, 2018

Accepted: January 8, 2019

Published: January 11, 2019

Copyright ( 2019 by author(s) and Scientific Research Publishing Inc. This work is licensed under the Creative Commons Attribution International License (CC BY 4.0).

http://creativecommons.org/licenses/by/4.0/

\begin{abstract}
The study of energetics, structural, the electronic and optical properties of $\mathrm{Ga}$ and As atoms substituted for doped germanane monolayers were studied by first-principles calculations based on density functional theory. Both of the two doping are thermodynamically stable. According to the band structure and partial density of the states, gallium is p-type doping. Impurity bands below the conduction band lead the absorption spectrum moves in the infrared direction. Arsenic doping has impurity level passing through the Fermi level and is $n$-type doping. The analysis of optical properties confirms the value of bandgap and doping properties.
\end{abstract}

\section{Keywords}

Germanane, Doping, Electronic Properties, Optical Properties, First-Principle

\section{Introduction}

Since the successful extraction of graphene by British scientists Andre Geim and Andre Geim using mechanical stripping in 2004, it has quickly led to the research and exploration of two-dimensional materials due to its excellent properties. In order to further enhance the application value of two-dimensional materials, it is important to improve the properties of materials by doing with graphene [1]-[14], transition-metal dichalcogenides [15] [16] [17] [18] [19] and other two-dimensional van der Waals material [20] [21] [22]. Germanane is a hydride similar to graphane with alternating hydrogen on either side of the germanium atom. Due to the direct band gap large electron mobility and the ability to control optoelectronic properties through covalent modification with surface ligands, it has received extensive attention in the field of two-dimensional materials [23] [24]. Experiments have shown that a system formed by 
doping element gallium of group III and element of arsenic group V into a germanane can be stably present. Doping the gallium and arsenic atoms into the precursor $\mathrm{CaGe}_{2}$ phase, in the process of using $\mathrm{HCl}$, remains intact in the lattice after the topotactic deintercalation to form germanane [25] [26] [27] [28] [29]. This work demonstrates that extrinsic doping with $\mathrm{Ga}$ and $\mathrm{As}$ is a viable pathway towards accessing stable electronic behavior in graphane analogues of germanium. But $\mathrm{Ga}$ and As doping in germanane have not been systematic research in theoretical calculation. In this work, we have studied thermodynamic stability, structural and electronic properties of gallium and arsenic defects in a germanane monolayer using a GGA (generalized gradient approximation) hybrid density functional theory approach. This study fine tunes the band gap of germanane through the foreign atom doping as well as via changing the charge state of the defect.

\section{Calculation Method}

The first principle calculation is implemented in CASTEP (Cambridge Serial Total Energy Package) code [30] [31] [32] by plane wave pseudopotential method based on density functional theory. In the calculation, pristine germanane monolayer and its doping system were seen in Figures 1(a)-(c). and the vacuum is $20 \AA$. In order to make the structure more stable, different replacement methods were used for gallium and arsenic atoms. Because the outermost electrons of gallium have only three electrons. We replace the germanium atom with a gallium atom and remove the hydrogen atom corresponding to the replaced germanium atom. However, there are five outermost electrons in the arsenic atom, so we only replaced the germanium atom with a arsenic atom, and did not remove the corresponding hydrogen atom. In the calculation process, the ions-electrons and exchange-correlation interaction are processed using norm-conserving pseudopotential and Perdew, Burke and Ernzerhof (PBE) functionals in GGA, respectively. In order to obtain an accurate optimized structure of germanane, a cutoff energy of $400 \mathrm{eV}$ is used to ensure total energy and stress convergence. In the process of optimization, the specific parameters are set as follows. The total energy is $5.0 \times 10^{-6} \mathrm{eV} /$ atom. The maximum stress, pressure, displacement, respectively $0.02 \mathrm{GPa}, 5.0 \times 10^{-4} \AA, 0.01 \mathrm{eV} / \AA$. In addition, the calculation of self-consistent field (SCF) is kept within the energy convergence criterion of $5 \times$ $10^{-6} \mathrm{eV} /$ atom. The Brillouin zone was $3 \times 3 \times 1 \mathrm{k}$-point mesh [33].

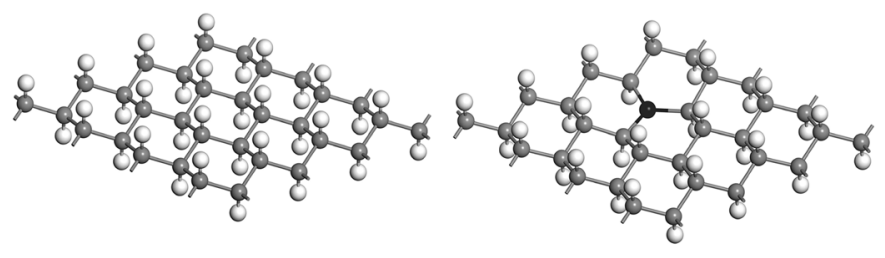

(a)pristine germanane
(b)Ga doped germanane

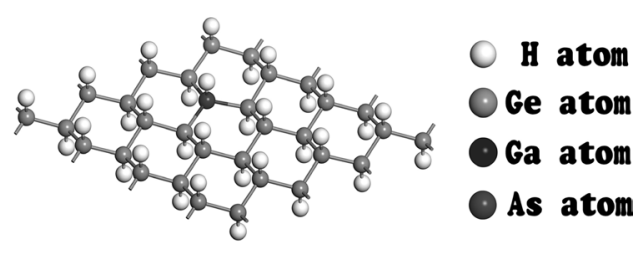

(c)As doped germanane

Figure 1. Top view of the supercell used in calculation: (a) Pristine germanane monolayer, (b) and (c) are gallium and arsenic defected germanane monolayer $(4 \times 4 \times 1)$. 
To assess the stabilities of nanostructures, we calculate the formation energy $E_{F}$ as follows:

$$
\begin{gathered}
E_{\text {form }}(X)=E_{X}-E_{\text {germanium }}+\mu_{\mathrm{Ge}}+\mu_{\mathrm{H}}-\mu_{\mathrm{Ga}} \\
E_{\text {form }}(X)=E_{X}-E_{\text {germanium }}+\mu_{\mathrm{Ge}}-\mu_{\mathrm{As}}
\end{gathered}
$$

The first two terms $E_{X}$ and $E_{\text {germanium }}$ are the total energies of $\mathrm{Ga}$ or As doped germanane and pristine germanium respectively. The $\mu_{\mathrm{Ge}}$ and $\mu_{\mathrm{H}}$ terms are the chemical potentials of the host $\mathrm{Ge}$ atom (obtained as the total energy per $\mathrm{Ge}$ atom from the unit cell of germanane monolayer) and $\mathrm{H}$ atom (obtained as the total energy per $\mathrm{H}$ atom from the hydrogen molecule), whereas the $\mu_{X}$ term is the chemical potential of the $\mathrm{Ga}$ defect (obtained as the total energy per $\mathrm{Ga}$ atom from face centered cubic Ga structure) or As defect (obtained as the total energy per As atom from the corrugate hexagonal structure structure) respectively. The calculation results show that the formation energies of gallium and arsenic doping are $-2.01 \mathrm{eV}$ and $-0.63 \mathrm{eV}$, respectively. This means that the two structures are thermodynamically stable.

\section{Results and Discussion}

\subsection{Structure Properties}

The Ge-Ge bond length ( $\left.d_{\text {Ge-Ge }}\right)$, the lattice parameter $(a)$, and the buckled height ( $\Delta z$, show in Figure 2) in the optimized structure of the pristine germanane monolayer are $2.455 \AA, 4.060 \AA$ and $0.730 \AA$, respectively. These values are in good agreement with experimental results and theoretical calculations [34].

After the doping of gallium, the structure and symmetry distort much. The length of the bond between the gallium atom and the neighboring germanane atom is $2.455 \AA, 2.455 \AA, 2.458 \AA$ respectively. The nearby Ge-Ge bond length also have changed in the range of $-0.3 \AA$ to $+0.3 \AA$. Gallium defect also causes large changes in corrugate, buckled height $\Delta z_{\mathrm{Ge}-\mathrm{Ge}}=0.730 \AA$ change to $\Delta z_{\mathrm{Ga}-\mathrm{Ge}}=0.047$ $\AA$. The hydrogen atom corresponding to the germanium atom interacting with gallium shifts away from the gallium atom. On the other hand, the doping of arsenic does not affect the structure and symmetry of the original germanane due to the remaining of the corresponding hydrogen atoms. The arsenic defected germanane monolayer is $\Delta z_{\mathrm{Ge}-\mathrm{As}}=0.728 \AA$ that is basically same as pristine germanane, and the original hexagonal structure is well maintained.

\subsection{Electronic Properties}

This section analyzes the mechanism of the change of the energy band width of pristine germanane and doping system. The band structure and distribution of the density are shown in Figure 3. Take the Fermi level as the zero point of energy.

Figure 3(a) show that pristine germanane has a direct bandgap and $E_{g}=1.080 \mathrm{eV}$, this is consistent with the theoretical calculation of the literature. The corresponding partial density of states of pristine germanane, as Figure $3(d)$, demonstrate that the 


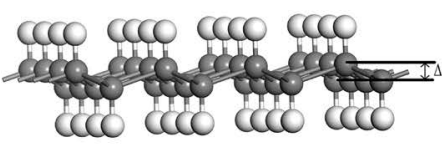

(a)pristine germanane

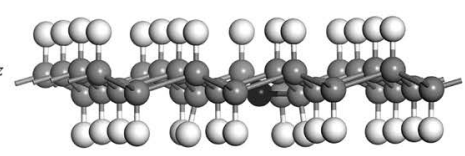

(b)Ga doped germanane

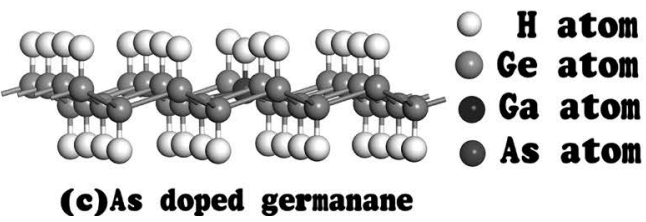

(c)As doped germanane

Figure 2. Side view of the supercell used in calculation: (a) pristine germanane monolayer (b) and (c) are gallium and arsenic defected germanane monolayer.
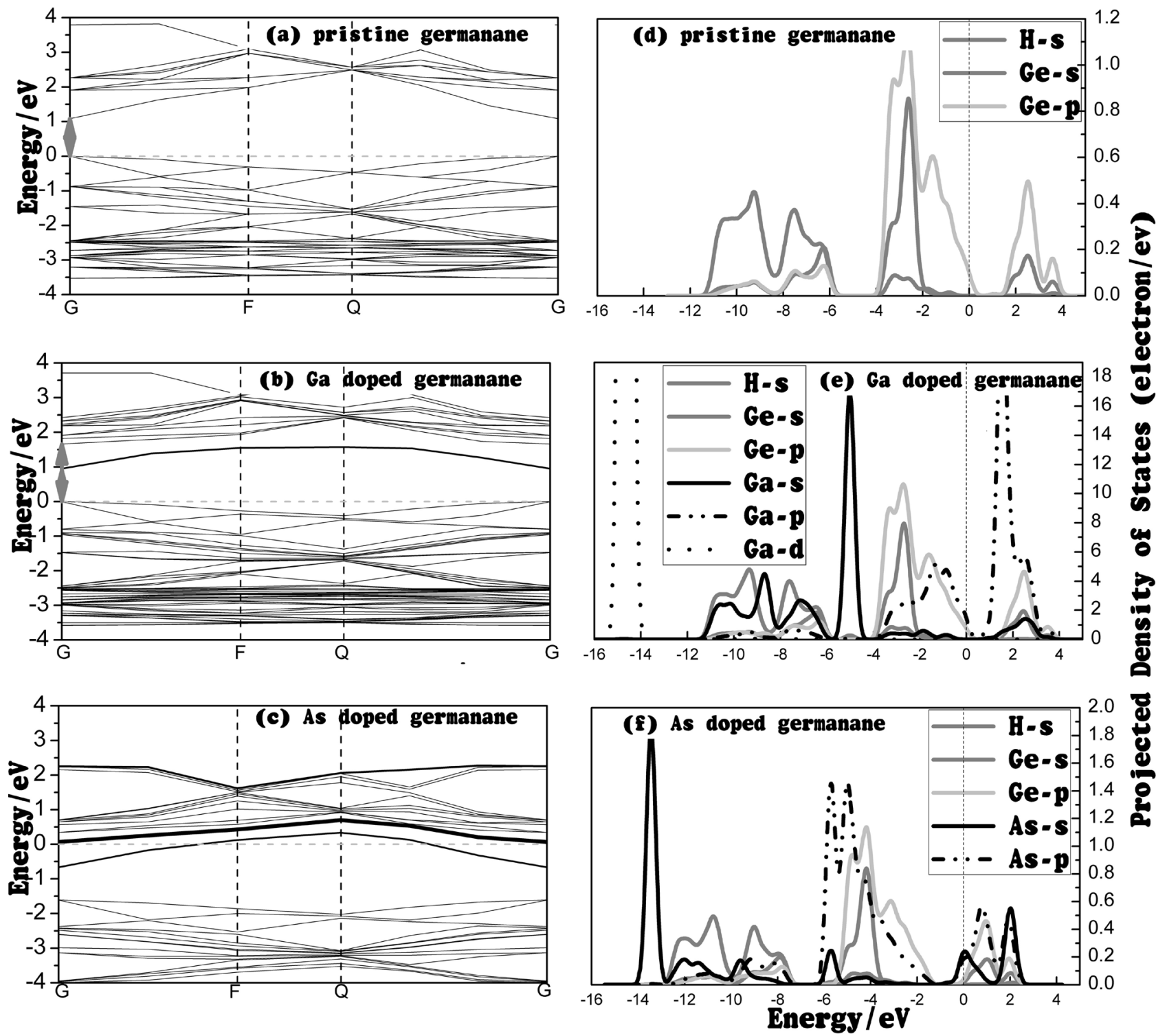

Figure 3. (a)-(c) Band structure of pristine germanane and its doping system; (d)-(f) Projected density of atates of pristine germanane and its doping system.

bottom of the conduction band is contributed by the Ge-4s and Ge-4p orbit, while the valence band of monolayer is derived from the contribution of $\mathrm{H}$-s, Ge-4s and Ge-4p orbit, but the top of valence is mainly contribute by $\mathrm{H}-\mathrm{s}$ and Ge-4p. Figure 3(b), Figure 3(e) give the electronic properties of gallium doped 
germanane. Bandgap increase to $E_{g}=1.663 \mathrm{eV}$. The projected density of states in Figure 3(e) show that Ga-4p contributes to the top of valence band, so it is a p-type doping. A unoccupied Ga-4p orbit impurity appears in the energy gap below conduction band. Due to the emergence of new energy levels, the the minimum energy of electrons from the valence band to the conduction band change to $0.949 \mathrm{eV}$. This energy value corresponds to the absorption edge position of the absorption spectrum of the gallium doping system. Figure $3(c)$ is the band structure of arsenic doped germanane. The valence band is contribute by $\mathrm{H}-\mathrm{s}, \mathrm{Ge}-4 \mathrm{p}$ and As-4p orbit, which has a strong hybrid coupling with each other. For arsenic atom has one more outermost electron than germanium atom, a occupied As-4p orbit impurity appears in the energy gap below conduction band. The impurity bands come across the Fermi level, so As is a n-type doping.

\subsection{Optical Properties}

In order to further explore its electronic properties, we also made specific calculations and data analysis on the optical properties of germanane. The study of electronic structures helps to make materials practically used in optoelectronic devices. Optical properties can be analyzed from the peaks and the intersection of the coordinates of the dielectric function. The dynamical dielectric function of germanane at arbitrary wave vector $q$ and frequency $\omega, \epsilon(q, \omega)$, is calculated in the self-consistent-field approximation. The corresponding optical constants of germanane and its doping structures can be analysis from the real and imaginary parts of the dielectric function. The absorption coefficient $n$, the complex refractive index and the reflectance $R$ of pristine germanane can be obtained by the Equations (4)-(6), respectively [35]. The dielectric function can be a relatively complete representation of the optical properties of the germane and its doped structure.

$$
\begin{gathered}
\varepsilon_{1}(\omega)=\frac{2 e^{2}}{\Omega \varepsilon_{0}} \sum\left|\left\langle\psi_{k}^{c}|u \cdot r| \psi_{k}^{v}\right\rangle\right| \delta\left(E_{k}^{c}-E_{k}^{v}-E\right) \\
\varepsilon_{2}(\omega)=1+\frac{2}{\pi} P \int_{0}^{\infty} \frac{\omega^{\prime} \varepsilon_{2}\left(\omega^{\prime}\right)}{\omega^{\prime 2}-\omega^{2}} \\
\alpha(\omega)=\sqrt{2}\left[\sqrt{\varepsilon_{1}^{2}(\omega)+\varepsilon_{2}^{2}(\omega)}-\varepsilon_{1}^{2}(\omega)\right]^{1 / 2} \\
n(\omega)=\frac{\sqrt{2}}{2}\left[\sqrt{\varepsilon_{1}^{2}(\omega)+\varepsilon_{2}^{2}(\omega)}+\varepsilon_{1}^{2}(\omega)\right]^{1 / 2} \\
k(\omega)=\frac{\sqrt{2}}{2}\left[\sqrt{\varepsilon_{1}^{2}(\omega)+\varepsilon_{2}^{2}(\omega)}-\varepsilon_{1}^{2}(\omega)\right]^{1 / 2} \\
R(\omega)=\left|\frac{\sqrt{\varepsilon(\omega)}-1}{\sqrt{\varepsilon(\omega)}+1}\right|^{2}
\end{gathered}
$$

Figure 4 depicts the dielectric function and the absorption coefficient of pristine germanane and its doping system for photon frequency up to $20 \mathrm{eV}$. The 

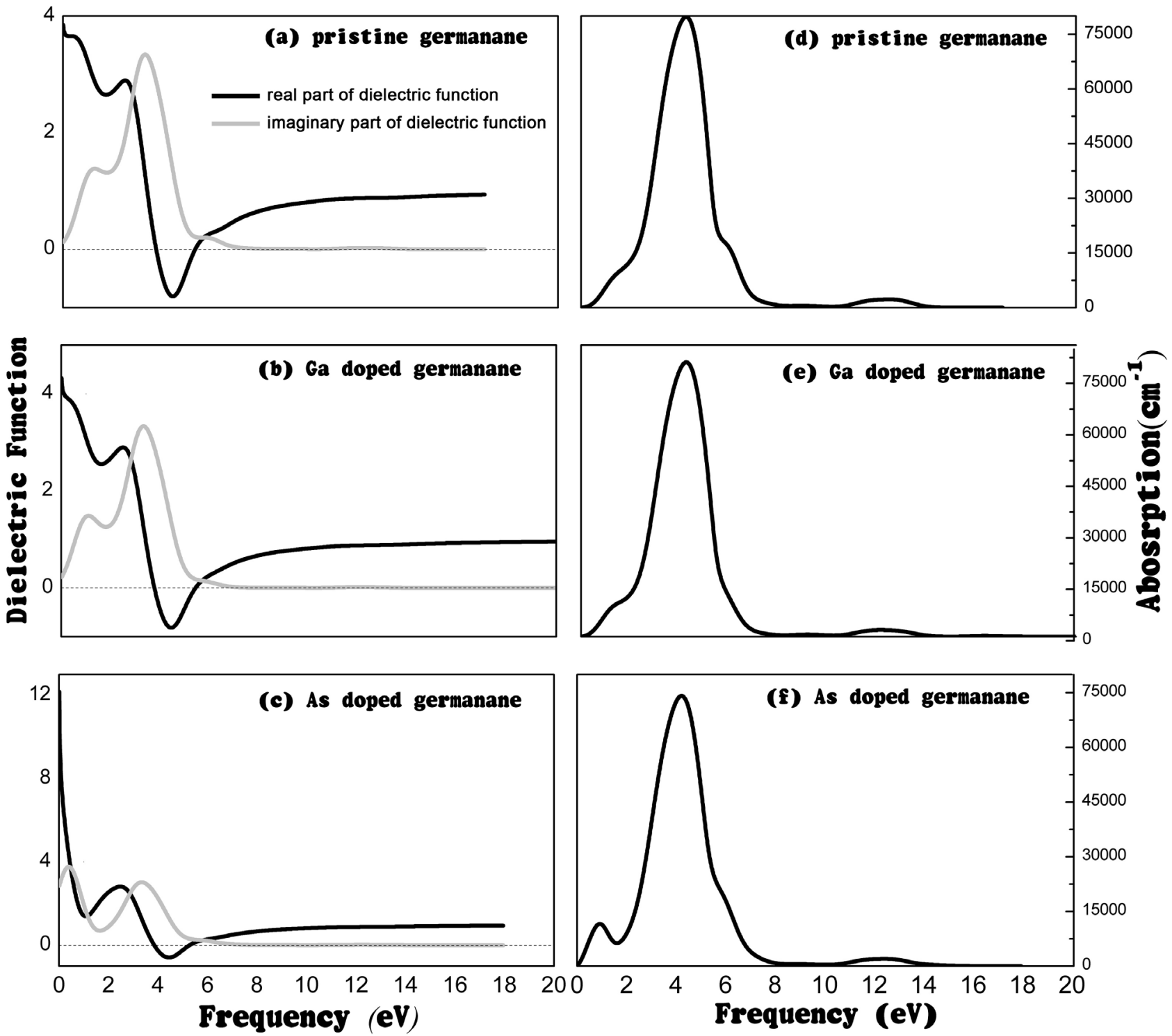

Figure 4. (a)-(c) Dielectric function of pristine germanane and its doping system; (d)-(f) Absorption coefficient of pristine and its doping system.

lines of black and grey in Figure 4 represent the real part and imaginary part of the dielectric function, respectively. As shown in Figure 4(a) and Figure 4(b) tendency of the dielectric function of pristine germanane and gallium doped germanane are consistent. It is observed that the static constant $\varepsilon_{1}(\omega)$ is close to 3.85 and 4.32 respectively. Curve Figure $4(\mathrm{a})$ /Figure 4 (b) mainly drops rapidly till $E=4.44 \mathrm{eV} / E=4.32 \mathrm{eV}$ and then increase slowly to $17 \mathrm{eV} / 22 \mathrm{eV}$. The imaginary part of dielectric function $\varepsilon_{2}(\omega)$ vanishes with the frequency $\omega=17 \mathrm{eV} / \omega=22 \mathrm{eV}$. It is noteworthy that the value $\varepsilon_{1}(\omega)>0$ and $\varepsilon_{2}(\omega)=0$ means the region is a transparent area. Refer to the absorption parameter as shown in Figure 4(a)/Figure 4(b), the optical absorption edge is about 1.080 $\mathrm{eV} / 0.949 \mathrm{eV}$ that is corresponding to the band gap of pristine germanane and gallium doped germanane in our calculation. Figure 4 (c) show the static con- 
stant $\varepsilon_{1}(\omega)$ is close to 12.2 , dielectric constant is a measure of the polarizability of a material. It shows that the arsenic doping has a stronger electrode forming ability.

\section{Conclusion}

Germanane monolayer was doped with gallium atom or arsenic atom. The Ga defect can be integrated within a germanane monolayer at a relative low formation energy, without major structural distortions and symmetry breaking. The As defect relaxes outward of the monolayer and breaks the symmetry. The density of states plots indicate that Ga doped germanane monolayer is p-type doping, whereas the As, which has a extra outermost electron, is n-type doping. The optical properties of $\mathrm{Ga}$ and As doping were also examined, the result demonstrates that As doping has static dielectric constants $\varepsilon_{1}(\omega)$ close to12 which means a stronger polarization ability. The results of this study will be of great guiding significance for the practical application.

\section{Conflicts of Interest}

The authors declare no conflicts of interest regarding the publication of this paper.

\section{References}

[1] Novoselov, K.S., et al. (2005) Two-Dimensional Gas of Massless Dirac Fermions in Graphene. Nature, 438, 197.

[2] Jiang, S., Arguilla, M.Q., Cultrara, N.D. and Goldberger, J.E. (2014) CovalentlyControlled Properties by Design in Group IV Graphane Analogues. Accounts of Chemical Research, 48, 144-151. https://doi.org/10.1021/ar500296e

[3] Garcia, J.C., de Lima, D.B., Assali, L.V. and Justo, J.F. (2011) Group IV Grapheneand Graphane-Like Nanosheets. The Journal of Physical Chemistry C, 115, 13242 13246. https://doi.org/10.1021/jp203657w

[4] Madhushankar, et al. (2017) Electronic Properties of Germanane Field-Effect Transistors. 2D Materials, 4, 021009.

[5] Vogg, G., Brandt, M.S. and Stutzmann, M. (2000) Polygermyne-A Prototype System for Layered Germanium Polymers. Advanced Materials, 12, 1278-1281. https://doi.org/10.1002/1521-4095(200009)12:17<1278::AID-ADMA1278>3.0.CO;2-Y

[6] Vogt, P., et al. (2012) Silicene: Compelling Experimental Evidence for Graphenelike Two-Dimensional Silicon. Physical Review Letters, 108, 155501. https://doi.org/10.1103/PhysRevLett.108.155501

[7] Wei, W., Dai, Y., Huang, B. and Jacob, T. (2013) Many-Body Effects in Silicene, Silicane, Germanene and Germanane. Physical Chemistry Chemical Physics, 15, 8789-8794. https://doi.org/10.1039/c3cp51078f

[8] Bonaccorso, F., et al. (2015) Graphene, Related Two-Dimensional Crystals, and Hybrid Systems for Energy Conversion and Storage. Science, 347, 1246501. https://doi.org/10.1126/science.1246501

[9] De Padova, P., et al. (2013) Evidence of Dirac Fermions in Multilayer Silicene. Applied Physics Letters, 102, 163106. https://doi.org/10.1063/1.4802782 
[10] Zhang, R.W., et al. (2015) Ethynyl-Functionalized Stanene Film: A Promising Candidate as Large-Gap Quantum Spin Hall Insulator. New Journal of Physics, 17, 083036. https://doi.org/10.1088/1367-2630/17/8/083036

[11] Zhang, R.W., et al. (2016) Room Temperature Quantum Spin Hall Insulator in Ethynyl-Derivative Functionalized Stanene Films. Scientific Reports, 6, 18879. https://doi.org/10.1038/srep18879

[12] Shin, B.G. and Oh, D.H. (2012) Influence of Orbital Hybridization on the Atomic and Electronic Structures in Hydrogenated Monolayer Graphene. Journal of the Korean Physical Society, 60, 816-820. https://doi.org/10.3938/jkps.60.816

[13] Abrahams, E., Anderson, P.W., Licciardello, D.C. and Ramakrishnan, T.V. (1979) Scaling Theory of Localization: Absence of Quantum Diffusion in Two Dimensions. Physical Review Letters, 42, 673. https://doi.org/10.1103/PhysRevLett.42.673

[14] Kapitanova, O.O., Panin, G.N., Baranov, A.N. and Kang, T.W. (2012) Synthesis and Properties of Graphene Oxide/Graphene Nanostructures. Journal of the Korean Physical Society, 60, 1789-1793. https://doi.org/10.3938/jkps.60.1789

[15] Radisavljevic, B. and Kis, A. (2013) Mobility Engineering and a Metal-Insulator Transition in Monolayer $\mathrm{MoS}_{2}$. Nature Materials, 12, 815. https://doi.org/10.1038/nmat3687

[16] Radisavljevic, B., Radenovic, A., Brivio, J., Giacometti, I.V. and Kis, A. (2011) Single-Layer $\mathrm{MoS}_{2}$ Transistors. Nature Nanotechnology, 6, 147.

https://doi.org/10.1038/nnano.2010.279

[17] Qian, X., Liu, J., Fu, L. and Li, J. (2014) Quantum Spin Hall Effect in TwoDimensional Transition Metal Dichalcogenides. Science, 346, 1344-1347. https://doi.org/10.1126/science.1256815

[18] Mak, K.F., Lee, C., Hone, J., Shan, J. and Heinz, T.F. (2010) Atomically Thin $\mathrm{MoS}_{2}$ : A New Direct-Gap Semiconductor. Physical Review Letters, 105, Article ID: 136805. https://doi.org/10.1103/PhysRevLett.105.136805

[19] Elias, D.C., et al. (2009) Control of Graphene's Properties by Reversible Hydrogenation: Evidence for Graphane. Science, 323, 610-613. https://doi.org/10.1126/science.1167130

[20] Arguilla, M.Q., et al. (2017) EuSn2As2: An Exfoliatable Magnetic Layered ZintlKlemm Phase. Inorganic Chemistry Frontiers, 4, 378-386. https://doi.org/10.1039/C6QI00476H

[21] Arguilla, M.Q. (2016) $\mathrm{NaSn}_{2} \mathrm{As}_{2}$ : An Exfoliatable Layered van der Waals Zintl Phase. ACS Nano, 10, 9500-9508. https://doi.org/10.1021/acsnano.6b04609

[22] Geim, A.K. and Grigorieva, I.V. (2013) Van der Waals Heterostructures. Nature, 499, 419. https://doi.org/10.1038/nature12385

[23] Freitas, R.R., et al. (2015) Topological Insulating Phases in Two-Dimensional Bismuth-Containing Single Layers Preserved by Hydrogenation. The Journal of Physical Chemistry C, 119, 23599-23606. https://doi.org/10.1021/acs.jpcc.5b07961

[24] Carrete, J., Li, W., Lindsay, L., Broido, D.A., Gallego, L.J. and Mingo, N. (2016) Physically Founded Phonon Dispersions of Few-Layer Materials and the Case of Borophene. Materials Research Letters, 4, 204-211. https://doi.org/10.1080/21663831.2016.1174163

[25] Bianco, E., Butler, S., Jiang, S., Restrepo, O.D., Windl, W. and Goldberger, J.E. (2013) Stability and Exfoliation of Germanane: A Germanium Graphane Analogue. Acs Nano, 7, 4414-4421. https://doi.org/10.1021/nn4009406

[26] Jiang, S., Bianco, E. and Goldberger, J.E. (2014) The Structure and Amorphization 
of Germanane. Journal of Materials Chemistry C, 2, 3185-3188.

https://doi.org/10.1039/c3tc32489c

[27] Jiang, S., et al. (2014) Improving the Stability and Optical Properties of Germanane via One-Step Covalent Methyl-Termination. Nature Communications, 5, Article No. 3389. https://doi.org/10.1038/ncomms4389

[28] Jiang, S., et al. (2016) Tailoring the Electronic Structure of Covalently Functionalized Germanane via the Interplay of Ligand Strain and Electronegativity. Chemistry of Materials, 28, 8071-8077.

https://doi.org/10.1021/acs.chemmater.6b04309

[29] Cultrara, N.D., et al. (2017) Group-13 and Group-15 Doping of Germanane. Beilstein Journal of Nanotechnology, 8, 1642-1648. https://doi.org/10.3762/bjnano.8.164

[30] Segall, M.D., et al. (2002) First-Principles Simulation: Ideas, Illustrations and the CASTEP Code. Journal of Physics. Condensed Matter, 14, 2717-2744. https://doi.org/10.1088/0953-8984/14/11/301

[31] Schwarz, K., Blaha, P. and Madsen, G.K. (2002) Electronic Structure Calculations of Solids Using the WIEN2k Package for Material Sciences. Computer Physics Communications, 147, 71-76. https://doi.org/10.1016/S0010-4655(02)00206-0

[32] Fischer, T.H and Almlof, J. (1992) General Methods for Geometry and Wave Function Optimization. The Journal of Physical Chemistry, 96, 9768-9774. https://doi.org/10.1021/j100203a036

[33] Chadi, D.J. (1977) Special Points for Brillouin-Zone Integrations. Physical Review B Condensed Matter, 16, 5188-5192. https://doi.org/10.1103/PhysRevB.16.1746

[34] Luo, X. and Zurek, E. (2015) Crystal Structures and Electronic Properties of Single-Layer, Few-Layer, and Multilayer GeH. The Journal of Physical Chemistry $C$, 120, 793-800. https://doi.org/10.1021/acs.jpcc.5b11770

[35] Shen, X.C. (1992) The Spectrum and Optical Property of Semiconductor, Optical Properties of Semiconductors. 